\title{
Addendum to: complete electromagnetic multipole expansion including toroidal moments Rev. Mex. Fis E 52 (2006) 188-197
}

\author{
E. Ley Koo \\ Instituto de Física, Universidad Nacional Autónoma de México, Ciudad de México, México.
}

A. Góngora T.

Formely of Northeastern University, Boston Ma.

H. Torres-Bustamante

Instituto de Física, Universidad Nacional Autónoma de México, Ciudad de México, México.

Received 29 January 2020; accepted 14 February 2020

\begin{abstract}
This Addendum reports exact and complete solutions for the electromagnetic field of poloidal currents uniformly distributed on spherical toroidal surfaces, which has been a pending task of Sec. 3 in [1]. This result is important by itself, and also because it allows the identification of new and alternative solutions and the reasons behind them.
\end{abstract}

Keywords: Electromagnetic radiation; multipole expansion; electric; magnetic; toroidal moments.

PACS: 41.20.Jb.

The writing of this Addendum is motivated by the citations of our work [1] in the reports on the observation of toroidal dipole interactions in metamaterials $[2,3]$ and nanomaterials and molecules [4], as recently reviewed in RMF [5]. In fact, the emphasis in [1] was on the distinction between the familiar electric and magnetic multipole moments and the new toroidal ones, with their respective sources of longitudinal, toroidal, and poloidal current distributions. Notice the last sentence at the end of Sec. 3.2. "For the sake of space, the complete expressions for the electric intensity and magnetic induction fields are not written out explicitly".

This Addendum reports the final form of the toroidal magnetic induction field, reminding the readers that: the current is uniformly distributed on meridian loops, with pairs of concentric circular arcs connected with pairs of radial seg-
DOI: https://doi.org/10.31349/RevMexFisE.17.138

ments, forming a toroidal surface, Eq. (23); the outgoing wave Green function in its spherical multipole expansion is given by Eq. (8); and the magnetic induction field is evaluated via their integral of Eq. (10). The solutions outside the toroidal surface, including its hole, involve outgoing waves, and the solutions inside are well-behaved.

To begin, the second angular integral in Eq. (29) involves the Legendre Polynomials of associativity $m=1$, and their corresponding eigenvalues with the result

$$
-\ell(\ell+1)\left[P_{\ell}^{1}\left(\cos \theta_{1}\right)-P_{\ell}^{1}\left(\cos \theta_{2}\right)\right],
$$

which is different from the integral of Eq. (27).

Next, we write the magnetic induction field inside the toroid involving the radial integrals over $h_{\ell}^{(1)}\left(k r^{\prime}\right)$ at the surface:

$$
\begin{aligned}
& \vec{B}^{i}\left(a<r<b ; \theta_{1}<\theta<\theta_{2} ; 0<\varphi<2 \pi\right)=-\frac{N I}{c} 4 \pi i k \hat{\varphi} e^{i \omega t} \sum_{\ell} N_{\ell, 1}^{2} P_{\ell}^{1}(\cos \theta) j_{\ell}(k r)\left\{\left[P_{\ell}\left(\cos \theta_{1}\right)-P_{\ell}\left(\cos \theta_{2}\right)\right]\right. \\
& \left.\quad \times\left.\left[\frac{d}{d r^{\prime}}\left(r^{\prime} h_{\ell}^{(1)}\left(k r^{\prime}\right)\right)\right]\right|_{r^{\prime}=a} ^{r^{\prime}=b}-\ell(\ell+1)\left[P_{\ell}^{1}\left(\cos \theta_{1}\right)-P_{\ell}^{1}\left(\cos \theta_{2}\right)\right]\left[\int_{a}^{b} d r^{\prime} \frac{h_{\ell}^{(1)}\left(k r^{\prime}\right)}{r^{\prime}}\right]\right\} .
\end{aligned}
$$

Then, the magnetic induction field outside the toroid involves the radial integral over the $j_{\ell}\left(k r^{\prime}\right)$ at the surface:

$$
\begin{aligned}
& \vec{B}^{e}(r ; \theta ; \varphi)=-\frac{N I}{c} 4 \pi i k \hat{\varphi} e^{i \omega t} \sum_{\ell} N_{\ell, 1}^{2} P_{\ell}^{1}(\cos \theta) h_{\ell}^{(1)}(k r) \\
& \times\left\{\left.\left[P_{\ell}\left(\cos \theta_{1}\right)-P_{\ell}\left(\cos \theta_{2}\right)\right]\left[\frac{d}{d r^{\prime}}\left(r^{\prime} j_{\ell}\left(k r^{\prime}\right)\right)\right]\right|_{r^{\prime}=a} ^{r^{\prime}=b}-\ell(\ell+1)\left[P_{\ell}^{1}\left(\cos \theta_{1}\right)-P_{\ell}^{1}\left(\cos \theta_{2}\right)\right]\left[\int_{a}^{b} d r^{\prime} \frac{j_{\ell}\left(k r^{\prime}\right)}{r^{\prime}}\right]\right\} .
\end{aligned}
$$

The normal components of $\vec{B}$ inside and outside $\hat{r} \cdot \vec{B}$ and $\hat{\theta} \cdot \vec{B}$ are zero, and consequently continuous at the circular boundaries $r=a$ and $b$, and at the radial boundaries $\theta=\theta_{1}$ and $\theta_{2}$, satisfying Gauss' law in its boundary condition form. In contrast, 
its tangential components are discontinuous at the successive boundaries yielding the constant linear distribution currents, consistent with Ampere's law:

$$
\begin{aligned}
4 \pi \vec{K} & =\hat{r} \times\left. c\left(\vec{B}^{e}-\vec{B}^{i}\right)\right|_{r=a}=4 \pi \frac{N I \hat{\theta}}{a} \sum_{\ell} N_{\ell, 1}^{2}\left[P_{\ell}\left(\cos \theta_{1}\right)-P_{\ell}\left(\cos \theta_{2}\right)\right] P_{\ell}^{1}(\cos \theta), \\
4 \pi \vec{K} & =\hat{r} \times\left. c\left(\vec{B}^{e}-\vec{B}^{i}\right)\right|_{r=b}=4 \pi \frac{N I \hat{\theta}}{b} \sum_{\ell} N_{\ell, 1}^{2}\left[P_{\ell}\left(\cos \theta_{1}\right)-P_{\ell}\left(\cos \theta_{2}\right)\right] P_{\ell}^{1}(\cos \theta), \\
4 \pi \vec{K} & =\hat{\theta} \times\left. c\left(\vec{B}^{e}-\vec{B}^{i}\right)\right|_{\theta=\theta_{1}}=4 \pi i k N I \hat{r} \sum_{\ell} N_{\ell, 1}^{2} P_{\ell}^{1}\left(\cos \theta_{1}\right) P_{\ell}^{1}\left(\cos \theta_{1}\right) \ell(\ell+1) \\
& \times\left[\int d r^{\prime} \frac{j_{\ell}\left(k r^{\prime}\right)}{r^{\prime}} h_{\ell}^{(1)}(k r)-j_{\ell}(k r) \int d r^{\prime} \frac{h_{\ell}^{(1)}\left(k r^{\prime}\right)}{r^{\prime}}\right], \\
4 \pi \vec{K}= & \hat{\theta} \times\left. c\left(\vec{B}^{e}-\vec{B}^{i}\right)\right|_{\theta=\theta_{2}}=-4 \pi i k N I \hat{r} \sum_{\ell} N_{\ell, 1}^{2} P_{\ell}^{1}\left(\cos \theta_{2}\right) P_{\ell}^{1}\left(\cos \theta_{2}\right) \ell(\ell+1) \\
& \times\left[\int d r^{\prime} \frac{j_{\ell}\left(k r^{\prime}\right)}{r^{\prime}} h_{\ell}^{(1)}(k r)-j_{\ell}(k r) \int d r^{\prime} \frac{h_{\ell}^{(1)}\left(k r^{\prime}\right)}{r^{\prime}}\right] .
\end{aligned}
$$

The differences between the first terms of Eqs. (A.2) and (A.1) at the circular boundaries involve the product of the radial variable $k a$ and the Wronskian of the spherical Bessel functions $-i / k^{2} a^{2}$, and its counterpart for the external boundary, respectively, displaying the harmonic superposition of the meridian currents $P_{\ell}^{1}(\cos \theta)$, in opposite directions. Similar results were recognized in the analysis of the magnetic dipole and electric dipole radiations inside and outside a sphere [6]. On the other hand, the differences between the second terms of Eqs. (A.2) and (A.1) at the radial segment boundaries $\theta=\theta_{1}$ and $\theta_{2}$, involve the differences between the products of the respective spherical Bessel functions and the integral of its companion:

$$
\begin{aligned}
\ell(\ell+1) & {\left[\int d r^{\prime} \frac{j_{\ell}\left(k r^{\prime}\right)}{r^{\prime}} h_{\ell}^{(1)}(k r)\right.} \\
& \left.-j_{\ell}(k r) \int d r^{\prime} \frac{h_{\ell}^{(1)}\left(k r^{\prime}\right)}{r^{\prime}}\right]=-\frac{i}{k r} .
\end{aligned}
$$

Its common value, independent of $\ell$, can be proven from the Spherical Bessel differential equation. It also describes the inverse proportionality of the radial dependence of the linear current density. Notice that at the end of the radial segments where the connection with the circular arcs occur, the values become $-i / k a$ and $-i / k b$, common with those in the respective circular boundaries.

Additionally, the summations over the complete sets of orthonormal associated Legendre polynomials are identified as follows. We start with those of Eq. (A.4), identifying them with the Dirac Delta functions $\delta\left(\cos \theta-\cos \theta_{i}\right)=$ $\delta\left(\theta-\theta_{i}\right) / \sin \theta$ defining the radial segments where the currents are located; the presence of the factor $r \sin \theta$ as the radius of the parallel circle of the location is also recognized. Next, the coefficients in the ordinary Legendre polynomials in Eq. (A.3) the coefficients in the associated Legendre polynomials in Eq. (A.4), respectively, were obtained from the integrations in Eqs. (27-28); the corresponding summations are identified as the difference of the integrals of the Dirac delta functions in $\theta=\theta_{1}$ and $\theta=\theta_{2}$ with the value of 1 in the corresponding interval. The connection of these currents with that of Eq. (23) shows the consistency in the analysis. It is also important to point out the one-to-one correspondence for the respective multipoles, in the source and in the magnetic field.

The electric intensity field can be computed as the rotational of the toroidal magnetic induction field, Eqs. (A.1) and (A.2) using Faraday's Law, becoming a poloidal field sharing the same character as its current source Eq. (23).

The new results in this addendum are contained in Eqs. (A.1) and (A.2): exhibiting the multipole compositions of the toroidal magnetic induction field inside and outside, respectively, as well as in Eqs. (A.3) and (A.4), showing the multipole compositions of the poloidal currents in the respective spherical and conical components of the toroidal surface.

The solutions analyzed so far have been restricted to have $m=1$. The solutions with other values of $m$ are still open to be analyzed and applied. The internal solutions have been constructed with the ordinary spherical Bessel functions. More general solutions, as superpositions of $j_{\ell}(k r)$ and $n_{\ell}(k r)$, can and should also be considered.

The representations of the Dirac delta-function and Heaviside functions imply complete and orthonormal sets of functions, associated with specific boundary conditions. Our treatment deals with families of solutions for antennae and resonant cavity radiations with the same formalism. Ref. [6] shows that the latter correspond to a Dirichlet boundary condition problem, while the optimum solutions for the antennae 
correspond to a Neumann boundary condition problem. Nonoptimized solutions for the antennae come with the Robin boundary condition.

1. A. Góngora T. and E. Ley-Koo, Rev. Mex. Fis. E 52 (2006) 188197.

2. T. Kaelberer, V.A. Fedotov, N. Papasimakis, D.P. Tsai and N. Zheludev, Science 330 (2012) 1510-1512.

3. N. Papasimakis, V.A. Fedotov, V. Savinov, T. A. Raybould and N. Zheludev, Nature Materials (2016) 263-271. DOI: 10.1038/NMAT4563
4. N. Talebi, S. Guo and P. van Aken, Nanophotonics 7 (2018) 93-110.

5. E. Ley-Koo, Rev. Mex. Fis. 64 (2018) 326-366.

6. E. Ley-Koo, Ch. Esparza-López, H. Torres-Bustamante, Rev. Mex. Fis. E. 64 (2018) 139-148. 